In-vitro-Studie bestätigt Prinzip der GAG-Ersatztherapie mit Chondroitinsulfat

\section{- Chondroitinsulfat ist der entscheidende} Baustein der Glykosaminoglykan-(GAG)Schicht in der Blasenwand. Ist die GAGSchicht geschädigt, können reizende Substanzen aus dem Urin bis in tiefer gelegene Schichten der Blasenwand vordringen. Als Folge kann es zu imperativen Harndrang, erhöhter Miktionsfrequenz und Schmerzen im kleinen Becken kommen. Ein Defekt der GAG-Schicht gilt als wahrscheinliche Ursache für die mit chronischen Zystitiden einhergehenden Symptome.

In einer aktuellen Studie untersuchten Janssen et al. unter anderem, in welchen Schichten der Blasenwand die unterschiedlichen GAGs zu finden sind [Janssen DAW et al. EAU 2012, abstr. 36845]. Hierzu wurden Biopsien aus unterschiedlich tiefen Gewebeschichten des Urothels gewonnen. Die Visualisierung der Verteilung der verschiedenen GAGs erfolgte mittels Agarose-Gelelektrophorese und Immunofluoreszenz-Assay. Die Ergebnisse waren folgende: Chondroitinsulfat ist das einzige GAG, das sich in der äußersten Blasenschutzschicht befindet. Heparan und
Dermatan waren nur in tiefer gelegenen Gewebsschichten nachweisbar, die keinen unmittelbaren Kontakt zum Urin haben. In einem zweiten Schritt wurde der Einfluss des jeweiligen GAG auf die Barrierefunktion des Urothels getestet. Dazu wurden primäre Urothelzellen kultiviert und die Barrierefunktion dieser Monolayer mittels trans-epitheliaren elektrischen Widerstand (TER) überprüft. Wie die Untersuchungen zeigten, trug lediglich Chondroitinsulfat signifikant zur Barrierefunktion bei.

Diese Resultate unterstreichen die große Relevanz des Chondroitinsulfats für die Barrierefunktion an der Grenzfläche zwischen Gewebe und Blaseninhalt. Klinische Daten und Studien bestätigen, dass Patienten mit chronischen Zystitiden, denen ein Defekt der GAG-Schicht zugrunde liegt, von der Instillationstherapie mit dem GAGErsatz Chondroitinsulfat (Gepan instill) profitieren.

Nach Informationen von

Pohl-Boskamp, Hohenlockstedt

\title{
Harnsäuresenkung: Interaktionspotenzial nicht unterschätzen
}

— Allopurinol gilt in Leitlinien der rheumatologischen Fachgesellschaften als Mittel der ersten Wahl bei der Harnsäuresenkung. Neben mangelnder Wirksamkeit und Unverträglichkeit können auch Arzneimittelinteraktionen ein Grund sein, besser Febuxostat (Adenuric $^{\oplus}$ ) einzusetzen.„Bei Febuxostat gibt es deutlich weniger Arzneimittelinteraktionen als bei Allopurinol. Daran sollte bei der Verordnung unbedingt gedacht werden", betonte Matthias Bastigkeit, Fachdozent für Pharmakologie, Geschendorf. Bei Febuxostat gebe es beispielsweise keine bekannten Interaktionen mit ACE-Hemmern wie Captopril, mit Immunsuppressiva wie Ciclosporin, mit Cumarinen oder mit den Antibiotika Amoxicillin und Ampicillin.

Für Allopurinol seien bei all diesen Medikamenten dagegen Interaktionen beschrieben, die teilweise sehr relevant seien, so Bastigkeit. Speziell zur Interaktion von Allopurinol und
ACE-Hemmern gebe es mittlerweile diverse Fallberichte, sodass laut Bastigkeit auf diese Kombination eher verzichtet werden sollte. Allopurinol sei mit Blick auf mögliche Arzneimittelinteraktionen auch deshalb als problematisch einzustufen, weil es überwiegend renal eliminiert wird. Bei Niereninsuffizienz droht damit eine Kumulation, die nicht nur das Risiko einer toxischen epidermalen $\mathrm{Ne}$ krolyse erhöht, sondern auch die Interaktionsgefahr. Eine Anpassung der Dosis von Allopurinol bei Nierenfunktionsstörungen sei deswegen obligat, so Bastigkeit, wohingegen die Febuxostat-Dosierung bei leichter bis mäßiger Niereninsuffizienz nicht angepasst werden müsse. Philipp Grätzel von Grätz

"GichtAkademie Fokus 2012 - Fortschritte in Diagnose und Therapie der Arthritis urica", Berlin, 9.-11. März 2012

Veranstalter: Berlin-Chemie, Berlin
Online-Fortbildung zu überaktiver Blase

Die Prävalenz der überaktiven Blase liegt in Deutschland bei $17 \%$, circa zehn Millionen Menschen sind betroffen und viele greifen lieber gleich zu Windeln und zu Vorlagen als mit ihrem Arzt zu sprechen. Dabei gibt es effektive Pharmakotherapien, die laut Studiendaten bis zu zwei Drittel der Patienten

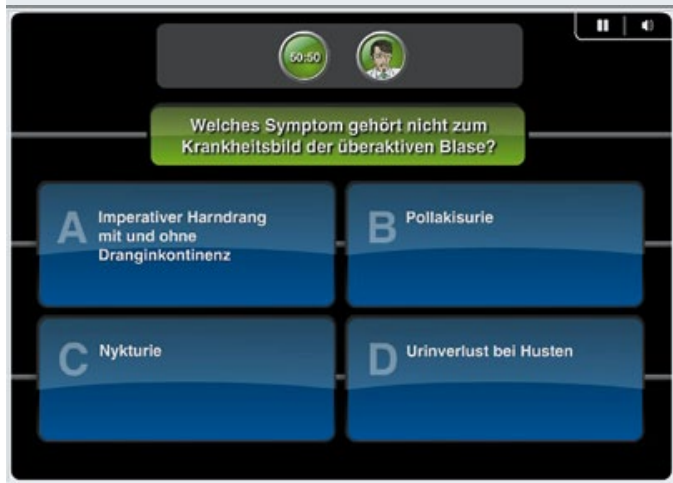

"trocken" werden ließen. Rund um das Thema Dranginkontinenz gibt es von Pfizer eine Online-Fortbildung (e-detailung). Auf www.toviaz-interaktiv.de erfährt der Nutzer in einem zehnminütigen interaktiven Wissenquiz alle Fakten zur überaktiven Blase und der Therapie, zum Beispiel mit Fesoterodinfumarat $\left(\right.$ Toviaz $\left.^{\oplus}\right)$. Die Teilnahme ist kostenlos, der Log-In kann über den Zugang von Springer Medizin erfolgen.

Nach Informationen von Pfizer, Berlin

\section{Neue Packungsgröße}

Das Farcosedan-SET ${ }^{\circledR}$ zur Prostata-Stanzbiopsie steht neben der bereits im Handel befindlichen 5er-Packung jetzt auch in einer patientengerechten 1er-Verpackungseinheit zur Verfügung. Damit hat Farco-Pharma auf die Rückmeldung von Urologen reagiert. Das sterile Set beinhaltet notwendige Medizinprodukte zur Durchführung einer transrektalen Prostatastanzbiopsie und kann durch die oberflächendesinfizierende Kombination der Substanzen Chlorhexidindigluconat und Methyl-4-hydroxybenzoat einen antiseptischen Schutzschild gegen vorhandene oder aszendierende Bakterien aufbauen [Sperling $\mathrm{H}$ et al. World J Urol 2002].

Nach Informationen von

Farco-Pharma, Köln 